\title{
Cardiac resynchronization sensitizes the sarcomere to calcium by reactivating GSK-3 $\beta$
}

\author{
Jonathan A. Kirk, ${ }^{1}$ Ronald J. Holewinski, ${ }^{1}$ Viola Kooij, ${ }^{1}$ Giulio Agnetti, ${ }^{1,2}$ \\ Richard S. Tunin, ${ }^{1}$ Namthip Witayavanitkul, ${ }^{3}$ Pieter P. de Tombe, ${ }^{3}$ \\ Wei Dong Gao, ${ }^{4}$ Jennifer Van Eyk, ${ }^{1}$ and David A. Kass ${ }^{1}$
}

\author{
1Division of Cardiology, Department of Medicine, Johns Hopkins University School of Medicine, Baltimore, Maryland, USA. \\ 2Department of Biomedical Science and Neuromotor Sciences, University of Bologna, Bologna, Italy. ${ }^{3}$ Department of Cell and Molecular Physiology, \\ Stritch School of Medicine, Loyola University Medical Center, Maywood, Illinois, USA. ${ }^{4}$ Department of Anesthesiology, \\ Johns Hopkins University, School of Medicine, Baltimore, Maryland, USA.
}

\begin{abstract}
Cardiac resynchronization therapy (CRT), the application of biventricular stimulation to correct discoordinate contraction, is the only heart failure treatment that enhances acute and chronic systolic function, increases cardiac work, and reduces mortality. Resting myocyte function also increases after CRT despite only modest improvement in calcium transients, suggesting that CRT may enhance myofilament calcium responsiveness. To test this hypothesis, we examined adult dogs subjected to tachypacing-induced heart failure for 6 weeks, concurrent with ventricular dyssynchrony $\left(\mathrm{HF}_{\mathrm{dys}}\right)$ or $\mathrm{CRT}$. Myofilament force-calcium relationships were measured in skinned trabeculae and/or myocytes. Compared with control, maximal calcium-activated force and calcium sensitivity declined globally in $\mathrm{HF}_{\mathrm{dys}}$; however, $\mathrm{CRT}$ restored both. Phosphatase $\mathrm{PP} 1$ induced calcium desensitization in control and CRT-treated cells, while $\mathrm{HF}_{\mathrm{dys}}$ cells were unaffected, implying that CRT enhances myofilament phosphorylation. Proteomics revealed phosphorylation sites on Z-disk and M-band proteins, which were predicted to be targets of glycogen synthase kinase-3 $\beta$ (GSK-3 $\beta$ ). We found that GSK-3 $\beta$ was deactivated in $\mathrm{HF}_{\mathrm{dys}}$ and reactivated by CRT. Mass spectrometry of myofilament proteins from $\mathrm{HF}_{\mathrm{dys}}$ animals incubated with GSK- $3 \beta$ confirmed GSK- $3 \beta$-dependent phosphorylation at many of the same sites observed with CRT. GSK-3 $\beta$ restored calcium sensitivity in $\mathrm{HF}_{\mathrm{dys}}$, but did not affect control or CRT cells. These data indicate that CRT improves calcium responsiveness of myofilaments following $\mathrm{HF}_{\mathrm{dys}}$ through GSK-3 $\beta$ reactivation, identifying a therapeutic approach to enhancing contractile function.
\end{abstract}

\section{Introduction}

For the past 30 years or more, the quest to develop treatments that directly improve systolic performance of the failing heart while also conferring long-term survival benefits has been met with disappointment (1). Agents that stimulate the $\beta 1$-adrenergic receptor or enhance cyclic AMP (e.g., PDE3 inhibitors) work in the short term, but have worsened chronic outcomes. This has led some to question whether safe and effective systolic therapy for the failing heart is an oxymoron (2). A counterexample that forced this conclusion to be reconsidered is the device treatment known as cardiac resynchronization therapy (CRT). CRT restores coordinate contraction in heart failure (HF) patients who also have ventricular dyssynchrony due to conduction abnormalities. Its improvement of systolic function occurs within 1 beat (3) and is associated with negligible or even reduced myocardial oxygen consumption, indicating improved chamber efficiency $(4,5)$. While dyssynchrony is an independent risk factor in patients with HF, successful CRT reduces mortality $(6,7)$, perhaps to levels even below those of $\mathrm{HF}$ patients without dyssynchrony $(8,9)$.

The effects of CRT were initially attributed to its impact on chamber mechanoenergetics (3). However, recent studies by our and other laboratories have revealed many changes at the cellular and molecular levels as well, and these present a unique profile for CRT unmatched by other HF treatments to date. For exam-

Conflict of interest: Jonathan A. Kirk and David A. Kass have a patent pending regarding the use of temporary dyssynchrony pacing to improve cardiac function in failing hearts.

Citation for this article: J Clin Invest. 2014;124(1):129-139. doi:10.1172/JCI69253. ple, marked heterogeneity of regional gene expression is reversed (10), the cytoprotective Akt pathway is stimulated and is coupled with reduced apoptosis $(11,12)$, calcium handling and associated ultrastructure of the sarcoplasmic reticulum improve (13-15), mitochondrial efficiency and ATP synthase activity are enhanced via a redox switch (16), ion channel function regulating the action potential is improved $(17,18)$, and resting as well as $\beta$-adrenergicstimulated myocyte function are markedly restored by a mechanism involving regulators of $\mathrm{G}$ protein signaling $(19,20)$. Interestingly, while CRT substantially improves resting myocyte function throughout the ventricle, this is not consistently matched by increases in the calcium transient $(17,20)$, suggesting CRT may also enhance myofilament responses to calcium. This is intriguing, as small molecule HF therapies that target the myofilament are of growing interest $(1,21-23)$.

Accordingly, we tested the hypothesis that myofilament function is depressed in dyssynchronous $\mathrm{HF}\left(\mathrm{HF}_{\mathrm{dys}}\right)$ and improved by CRT. Given that myofilament $\mathrm{Ca}^{2+}$ sensitivity is modulated by thinfilament protein phosphorylation (24-26) and prior data reveal CRT can modify several pertinent kinases and phosphatases (11), we speculated that CRT affects myofilament protein phosphorylation as an underlying mechanism. To investigate this, we studied a canine model combining tachypacing-induced HF with dyssynchrony or CRT $(19,20)$. Heart failure was induced by 6 weeks of rapid pacing. $\mathrm{HF}_{\mathrm{dys}}$ combined left bundle branch ablation with atrial tachypacing. For resynchronized HF (CRT), the same procedure was used for the initial 3 weeks, followed by rapid biventricular stimulation. These and other model modifications utilized in this study are summarized in Figure 1. Echocardiographic and hemodynamic 


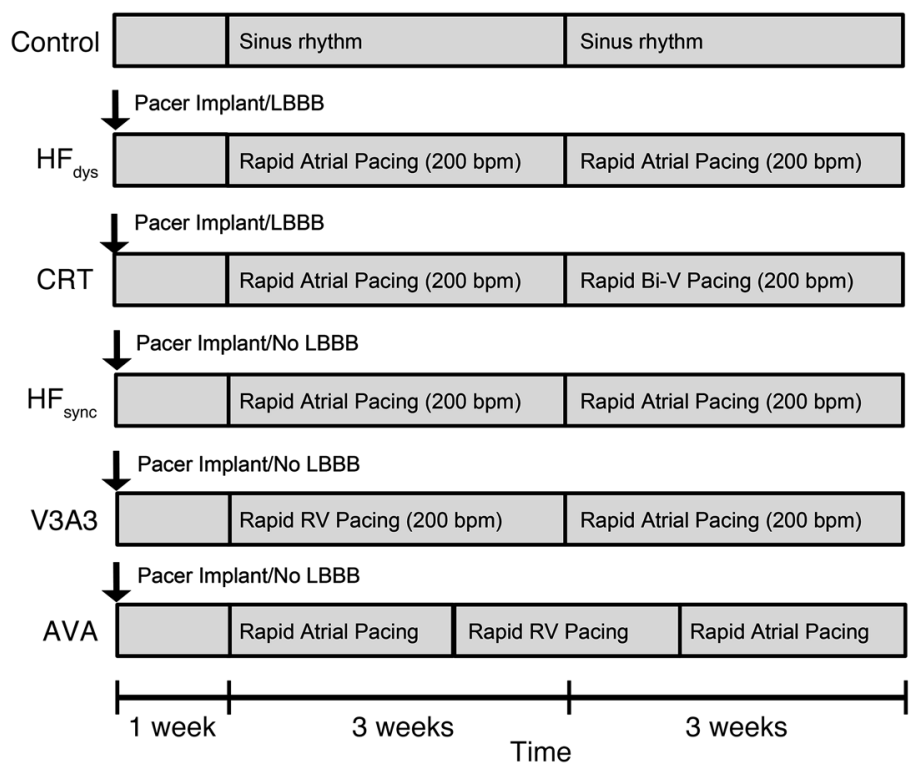

Figure 1

Timeline of pacing protocols in each of the canine models: control, $\mathrm{HF}_{\mathrm{dys}}, \mathrm{CRT}$, $\mathrm{HF}_{\text {sync, }}$, V3A3 (3 weeks RV pacing to induce dyssynchrony, 3 weeks atrial pacing to restore synchrony), and $\mathrm{AVA}\left(\mathrm{HF}_{\text {sync }}\right.$ with 2 weeks of induced transient dyssynchrony via $\mathrm{RV}$ pacing). The $\mathrm{HF}_{\text {dys }}$ and $C R T$ models receive $L B B B$ via radio-frequency ablation. After a 1-week recovery period from the pacemaker implant, each pacing protocol lasts 6 weeks before the animal is sacrificed.

features of these models have been reported $(19,20)$ and are summarized for the current set of animals in Supplemental Table 1 (supplemental material available online with this article; doi:10.1172/ JCI69253DS1). Trabecular and/or myocyte sarcomere function were assessed and proteomics used to identify phosphorylation changes linked to enhanced myofilament function from CRT.

\section{Results}

CRT globally restores myofilament function. Steady state force-calcium data for skinned myocytes from the LV lateral endocardium are shown in Figure 2A. Myofilament function was markedly depressed in $\mathrm{HF}_{\text {dys }}$, with maximum calcium-activated force $\left(\mathrm{F}_{\max }\right)$ declining nearly $50 \%$ compared with normal controls (controls: $37.9 \pm 3.5 \mathrm{mN} \cdot \mathrm{mm}^{-2}$, $n=15$ myocytes from 6 dogs, $\mathrm{HF}_{\mathrm{dys}}: 21.5 \pm 3.0 \mathrm{mN} \cdot \mathrm{mm}^{-2}, n=16$ myocytes from 6 dogs, $P<0.001$; Figure $2 \mathrm{~B})$. $\mathrm{HF}_{\mathrm{dys}}$ also exhibited $\mathrm{Ca}^{2+}$ desensitization as quantified by a rise in $\mathrm{EC}_{50}$ (controls: $2.34 \pm 0.13 \mu \mathrm{M}$, $\mathrm{HF}_{\text {dys }}: 3.04 \pm 0.17 \mu \mathrm{M}, P=0.003$; Figure $2 \mathrm{~B}$ ). CRT remarkably and completely restored both parameters of myofilament function to $\operatorname{normal}\left(\mathrm{F}_{\text {max }}: 40.3 \pm 2.60 \mathrm{mN} \cdot \mathrm{mm}^{-2}, \mathrm{EC}_{50}: 2.18 \pm 0.13 \mu \mathrm{M}, n=16\right.$ myocytes from 7 dogs, $P<0.001$ vs. $\mathrm{HF}_{\mathrm{dys}}, P=\mathrm{NS}$ vs. controls).

Contractile dyssynchrony with a left-bundle delay results in marked differences in regional stress (high in lateral wall due to early stretch/late contraction, lower in septum/RV with early contraction/ late stretch). To determine whether this influenced CRT modulation of myofilament function, we studied skinned trabeculae from the RV free wall (Figure 2, C and D) and single myocytes from the LV septum (Supplemental Figure 1). Both preparations revealed similarly depressed sarcomere function, as found in the $\mathrm{LV}$ free wall for $\mathrm{HF}_{\mathrm{dys}}$, and marked improvement with CRT. Thus, the precise nature of cyclical regional stress or strain temporal pattern was not a primary determinant of myofilament changes in either condition.
We next tested whether CRT alters crossbridge mechanoenergetics by simultaneously assessing isolated fiber bundle ATP utilization and force development. The ATP required to achieve a given crossbridge tension (tension cost) and the rate of force redevelopment upon sudden stress release (crossbridge kinetics, $k_{\mathrm{tr}}$ ) were similarly depressed in $\mathrm{HF}_{\text {dys }}$ and CRT (Figure 2, $\mathrm{E}$ and $\mathrm{F}$ ). The number of attached crossbridges during tension development, assessed by dynamic stiffness, was unchanged from control values by either $\mathrm{HF}_{\text {dys }}$ or CRT (Figure $2 \mathrm{G}$ ). Thus, CRT did not affect the mechanoenergetics of the crossbridge.

Importance of resynchronization. The recovery of myofilament function with CRT was not merely due to contraction being synchronous, as sarcomere function was also markedly depressed in HF that was always synchronous $\left(\mathrm{HF}_{\text {sync }}\right)$. As with $\mathrm{HF}_{\text {dys }}, \mathrm{F}_{\max }$ was much reduced in $\mathrm{HF}_{\text {sync }}$ $\left(21.08 \pm 3.22 \mathrm{mN} \cdot \mathrm{mm}^{-2}, P<0.001\right.$ vs. controls and CRT, $P=\mathrm{NS}$ vs. $\mathrm{HF}_{\mathrm{dys}}, n=15$ myocytes from 5 dogs; Supplemental Figure 2). However, whereas $\mathrm{HF}_{\mathrm{dys}}$ cells displayed a rise in $\mathrm{EC}_{50}$, those from $\mathrm{HF}_{\text {sync }}$ had a lower value (i.e., sensitized to $\mathrm{Ca}^{2+} ; 1.70 \pm 0.11 \mu \mathrm{M}, P=0.007,0.036,0.003$ vs. controls, $\mathrm{CRT}$, and $\mathrm{HF}_{\mathrm{dys}}$, respectively).

These data indicated that improved myofibrillar function required preexisting dyssynchrony that was subsequently reversed. To test this concept further, we modified the $\mathrm{HF}_{\text {sync }}$ model to include a 2-week period of dyssynchrony induced by RV pacing during weeks 3 and 4 (AVA model [atrial, ventricular, atrial pacing]) (19). Compared with $\mathrm{HF}_{\text {sync, }}$ AVA myocardium had a greater $F_{\max }$ (AVA: $23.4 \pm 1.9 \mathrm{mN} \cdot \mathrm{mm}^{-2}, n=10$ trabeculae from 5 dogs, $\mathrm{HF}_{\text {sync }}: 16.5 \pm 1.9, n=22$ trabeculae from 8 dogs, $P<0.05$ vs. $\mathrm{HF}_{\text {sync }}, P=\mathrm{NS}$ vs. controls) and $\mathrm{EC}_{50}$ returned to normal control values (Figure 3, A and B).

CRT involved direct ventricular myocardial stimulation that might have triggered improved myofibrillar function. To test this, we studied an alternative "CRT" model that used rapid RV free-wall pacing (no left bundle branch block [LBBB] was performed) to generate dyssynchrony and $\mathrm{HF}$ and then subsequently resynchronized the hearts by switching to rapid atrial pacing, avoiding the need for any direct ventricular stimulation. The results for this model (V3A3) were very similar to those with CRT (Figure 3, C and D), indicating direct pacemaker stimulation of the LV was not required. Altered maximum force is due to myocyte bypertrophy. The decline in $F_{\max }$ (normalized to myocyte cross-sectional area [CSA]) in both $\mathrm{HF}_{\text {dys }}$ and $\mathrm{HF}_{\text {sync }}$ could result from proteolytic cleavage of myofibrillar proteins such as troponin I (TnI) (27) or isoform switching (e.g., troponin $\mathrm{T}[\mathrm{TnT}$ ]; ref. 28). However, silver stained 1D gels and Western blots revealed neither protein cleavage products nor isoform switching in these models (Supplemental Figure 3). Normalization of force to CSA is generally performed, since one assumes bigger cells will have more force-generating units. Yet while control, $\mathrm{HF}_{\mathrm{dys}}$, and CRT cells had similar raw (nonnormalized) peak force, CSA differed markedly (Figure 4D). Specifically, $\mathrm{HF}_{\mathrm{dys}}$ had twice the CSA of controls, but this was reversed by CRT (controls: $251 \pm 36, \mathrm{HF}_{\mathrm{dys}}$ : $465 \pm 64$, CRT: $268 \pm 44 \mu \mathrm{m}^{2}, P<0.012$, $0.015 \mathrm{HF}_{\mathrm{dys}}$ vs. controls and CRT, respectively; Figure 4, A and B). To rule out potential selection bias in cells for which physiological data had been measured, we measured CSA from all cells in a given microscopic field ( $n=150$ myocytes from 3 dogs). This confirmed that $\mathrm{HF}_{\text {dys }}$ myocytes were enlarged and that CRT reversed this $(P<0.001$, Figure $4 C)$. In trabeculae, $F_{\max }$ was also depressed (Figure 2C), but CSA was similar among the models (Figure 4E). 
A

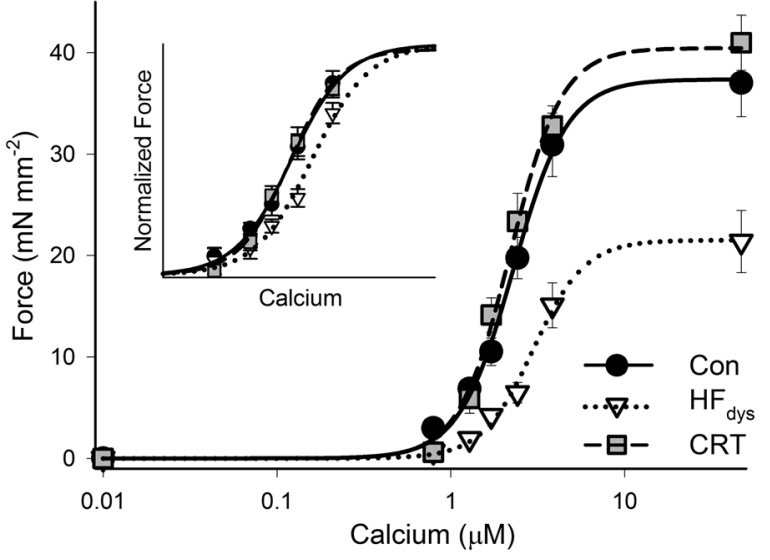

C
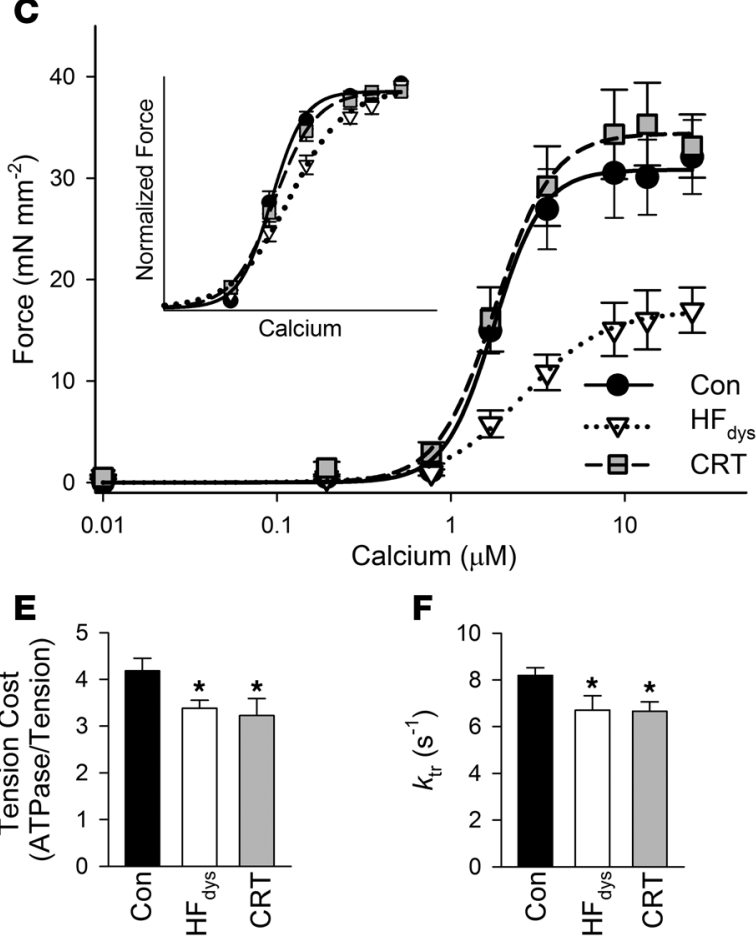

B

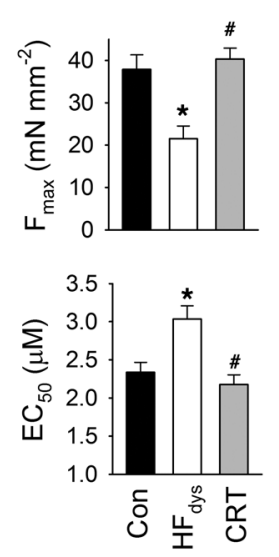

D
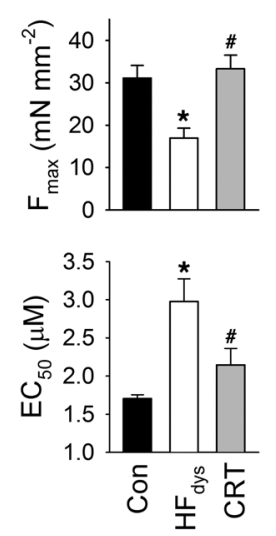

G

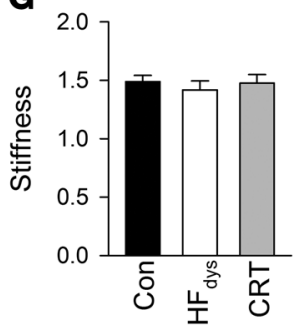

Figure 2

CRT globally restores myofilament function. (A) Force-calcium data and fitted curves for myocytes from the LV lateral wall for controls (black circles, $n=15$ myocytes from 6 dogs), $\mathrm{HF}_{\text {dys }}$ (white triangles, $n=16$ myocytes from 6 dogs), and CRT (gray squares, $n=16$ myocytes from 7 dogs). Inset: force normalized to $F_{\max }$ to visualize the change in calcium sensitivity. (B) $F_{\max }$ is decreased by approximately $50 \%$ in $\mathrm{HF}_{\text {dys }}$ and restored by CRT. $\mathrm{HF}_{\text {dys }}$ causes desensitization to calcium (increase in $\mathrm{EC}_{50}$ ), but is restored to control levels with CRT. (C) Force-calcium data and fitted curves for RV trabeculae muscles for control (black circles, $n=14$ muscles from 8 dogs), $\mathrm{HF}_{\text {dys }}$ (white triangles, $n=24$ muscles from 13 dogs), and CRT (gray squares, $n=18$ muscles from 10 dogs). Inset: force normalized to $F_{\max }$ to visualize the change in calcium sensitivity. (D) $F_{\max }$ and $E_{50}$ were altered in the RV to an extent similar to that in the LV. (E) Tension cost, the ratio of ATPase activity to developed tension, was reduced in both HF models, and not recovered by CRT. (F) Cross-bridge turnover dynamics $\left(k_{\mathrm{tr}}\right)$ were also reduced in the HF models and not restored by CRT. (G) Finally, stiffness was not affected in any of the groups. ${ }^{*} P<0.05$ vs. control; $\# P<0.05$ vs. $\mathrm{HF}_{\text {dys }}$ by 1-way ANOVA.
$\mathrm{Ca}^{2+}$ sensitization is mediated by myofilament phosphorylation. Figure $5 \mathrm{~A}$ displays example force-calcium data from RV trabeculae prior to and following incubation with the phosphatase PP1. Dephosphorylation did not change $F_{\max }$ in any group (Supplemental Figure $4 \mathrm{~A})$. However, $\mathrm{EC}_{50}$ increased (desensitized) in control $\left(\Delta \mathrm{EC}_{50}\right.$ : $1.09 \pm 0.22 \mu \mathrm{M}, n=6)$ and $\mathrm{CRT}\left(\Delta \mathrm{EC}_{50}: 0.70 \pm 0.23 \mu \mathrm{M}, n=5\right)$ trabeculae, but was unaltered in $\mathrm{HF}_{\mathrm{dys}}\left(\Delta \mathrm{EC}_{50}:-0.06 \pm 0.14 \mu \mathrm{M}\right.$, $n=5, P<0.003,0.04$ vs. controls and CRT, respectively; Figure $5 \mathrm{~B})$. $\mathrm{PP} 1$-induced calcium desensitization without a change in $\mathrm{F}_{\max }$ was also observed in V3A3 and AVA models, indicating phosphorylation was involved with their improved $\mathrm{Ca}^{2+}$ sensitivity as well (Supplemental Figure 4).

$\mathrm{Ca}^{2+}$ sensitivity is regulated by phosphorylation of the regulatory thin-filament proteins TnI, TnT, myosin-binding protein $\mathrm{C}$ (MyBPC), and myosin light chain 2 (MLC2). Table 1 lists the phosphorylation sites involved, their respective influence on $\mathrm{Ca}^{2+}$ sensitivity, and directional changes in each due to $\mathrm{HF}$ (if reported). Previous studies show that phosphorylation of TnI at S22/S23 (PKA) (29) and S43/S45 (PKC) $(30,31)$ desensitize, whereas phosphorylation at T144 (PKC) $(31,32)$ or S150 (AMPkinase, PAK1) $(33,34)$ increase sensitivity (the latter only reported in vitro). Overall, TnI phosphorylation declined similarly in $\mathrm{HF}_{\mathrm{dys}}$ and CRT (Figure 5C), and reduced S22/S23 targeting was directly confirmed by immunoblot (Supplemental Figure 5). This supports a $\mathrm{Ca}^{2+}$ sensitization effect (as opposed to desensitization observed in $\mathrm{HF}_{\text {dys }}$, e.g., Figure $2 \mathrm{~A}$ ). MyBPC phosphorylated at S273/S282/S302 (35) (by PKA and other kinases; ref. 36) or MLC2 phosphorylation at S15/S19 increases $\mathrm{Ca}^{2+}$-sensitivity (37). These posttranslational modifications (PTMs) declined to the same extent in both failure models (Figure 5, D and E) and so could not be responsible for the differences between $\mathrm{HF}_{\mathrm{dys}}$ and CRT. Finally, TnT phosphorylation at T206 (38) (PKC) or S278/ T287 (39) (Rho-kinase II) is linked to sensitization, but these sites were not altered in the models (Figure 5F). 
A

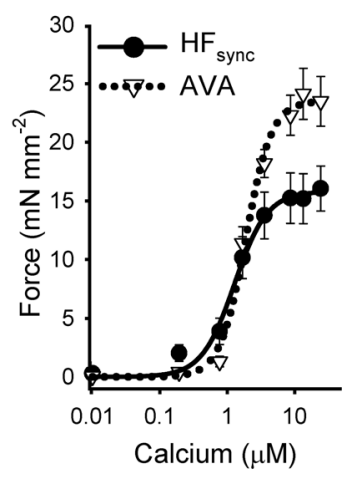

B

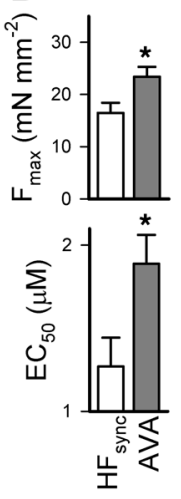

C

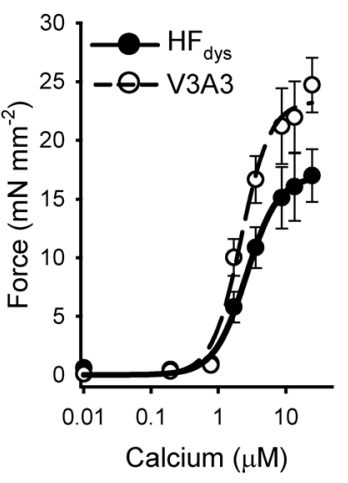

Figure 3

Myofilament function can be improved via a brief period of induced dyssynchrony. (A and $\mathbf{B}$ ) Both maximum force and calcium sensitivity are improved in the $\mathrm{HF}_{\text {sync }}$ model when the middle 2 weeks of the pacing protocol is made dyssynchronous (AVA model). (C and D) When dyssynchrony is induced via RV pacing (instead of radio frequency ablation of the left bundle branch) and resynchronization is restored by moving the pacing site to the atrium (V3A3), the effects of CRT are recapitulated. (C) Dyssynchrony caused a decrease in $F_{\max }$, which was increased by V3A3. (D) Dyssynchrony desensitized the myofilament response to calcium (increase in $\mathrm{EC}_{50}$ ), which was also reversed in the V3A3 model. ${ }^{*} P<0.05$ vs. $\mathrm{HF}_{\text {sync }}$ or $\mathrm{HF}_{\text {dys }}$.

The lack of differential changes in all of these sites was further confirmed by mass spectrometry (Supplemental Table 2) and/or 2D differential gel electrophoresis (Supplemental Figure 6).

Z-disk and M-band proteins are phosphorylated by CRT; role of GSK-3ß . Given that the known phospho-protein targets did not appear to be involved, we turned to mass spectrometry to broadly interrogate the myofibrillar subproteome. A total of 1187 phosphorylation sites on 166 myofilament and myofilament-associated proteins were detected, and CRT significantly altered 33 of these sites $(P<0.05$, quantified by normalized spectral counts; Table 2$)$. Based on the PP1 results, we focused on residues exhibiting increased phosphorylation with CRT, yielding 15 sites on 13 proteins (Table 2 and Supplemental Figures 7-21). Figure 6A summarizes this analysis. The majority of modified sites resided in proteins expressed in the Z-disk and $\mathrm{M}$-band regions. Though such pro-

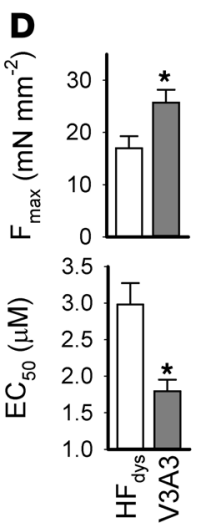
proteome obtained from $\mathrm{HF}_{\text {dys }}$ dogs teomic analysis can bias toward highabundance or high-molecular weight proteins (e.g., titin or obscurin), our analysis identified only a single site on each, supporting response specificity. We used in silico analysis of the individual phosphorylation sites (using NetPhosK and Human Protein Reference Database) to identify kinase consensus sequences based on the phosphorylation targets (Table 2) and found that glycogen synthase kinase$3 \beta$ (GSK-3 $\beta$ ) and p38 MAPK targeted the most sites. We previously found that p38 was activated by $\mathrm{HF}_{\text {dys }}$ and suppressed by CRT, whereas GSK-3 $\beta$ was less active in $\mathrm{HF}_{\mathrm{dys}}$ and activated by CRT (11). Therefore, we focused on the latter kinase as a potential contributor. To determine whether the phosphorylated residues increased by CRT were indeed targets of GSK-3 $\beta$, we performed a second mass spectrometry experiment. The myofilament subwas incubated with ${ }^{18} \mathrm{O}$-labeled ATP in the absence or presence of recombinant active GSK- $3 \beta$ for 30 minutes at $30^{\circ} \mathrm{C}$ and prepared for proteomic analysis. Table 3 lists the phosphorylated amino acid residues targeted by GSK-3 $\beta$ that were also found to increase with CRT. Four of the 7 GSK- $3 \beta$ sites revealed by in silico analysis were confirmed, and 4 additional sites altered by CRT but not predicted by bioinformatics were revealed as GSK- $3 \beta$ targets as well. This supports the notion that GSK-3 $\beta$ converts a subset of the $\mathrm{HF}_{\text {dys }}$ phospho-proteome into one similar to CRT.

To test whether GSK-3 $\beta$ myofilament phosphorylation could be responsible for the sensitizing affect, myofilament function was examined in $\mathrm{HF}_{\text {dys }}$ myocytes before and after exposure to recombinant active GSK-3 $\beta$ for 30 minutes. Treated cells exhibited a decline in $\mathrm{EC}_{50}$ similar to that observed with CRT $(P<0.001$ $\mathrm{HF}_{\text {dys }}$ vs. pretreatment, $P=\mathrm{NS}$ versus CRT; Figure 6, B and C). In
Figure 4

The apparent decrease in $F_{\max }$ is due to myocyte hypertrophy. (A) Skinned myocytes from controls, $\mathrm{HF}_{\text {dys }}$, and CRT. (B) CSA (calculated by measuring both width and depth and assuming an elliptical shape) was significantly increased in $\mathrm{HF}_{\text {dys, }}$, but restored to normal levels in CRT. (C) The unbiased approach also showed that $\mathrm{HF}_{\text {dys }}$ myocytes were hypertrophied, and this was reversed by CRT ( $n=150$ myocytes from 3 dogs per group). (D) $F_{\max }$ is typically normalized to CSA, but if it is not, the myocytes generate the same amount of force between the groups, indicating that the force producing capacity of the myocytes is not altered; they are just bigger. (E) There was no difference in trabeculae muscle size, despite the fact that a decrease in $F_{\max }$ was also observed. This suggests that there are fewer myocytes in $\mathrm{HF}_{\text {dys }}$ in a constant area of myocardium. ${ }^{*} P<0.05$ vs. control; ${ }^{P} P<0.05$ vs. $\mathrm{HF}_{\text {dys }}$ by 1 -way ANOVA.
A
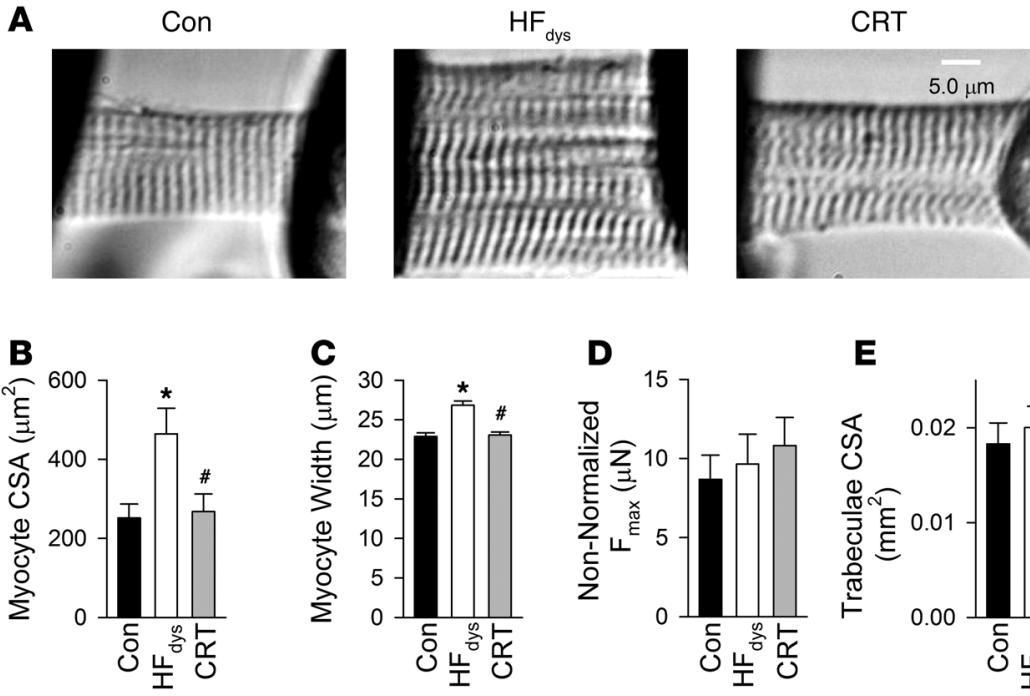

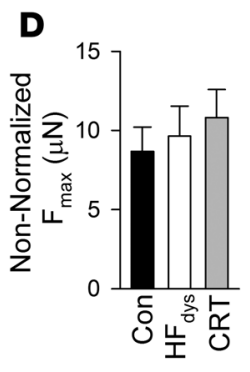

E

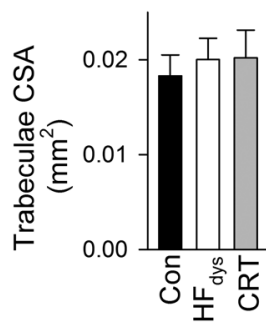


A

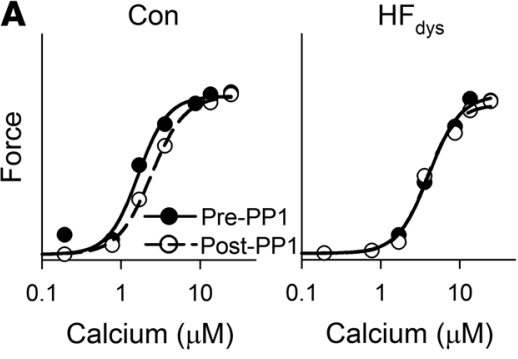

CRT

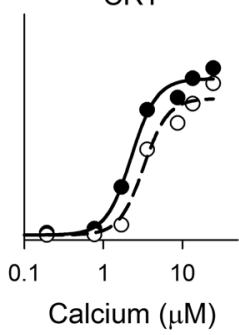

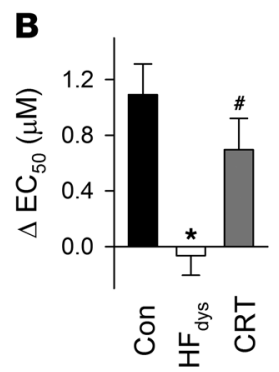
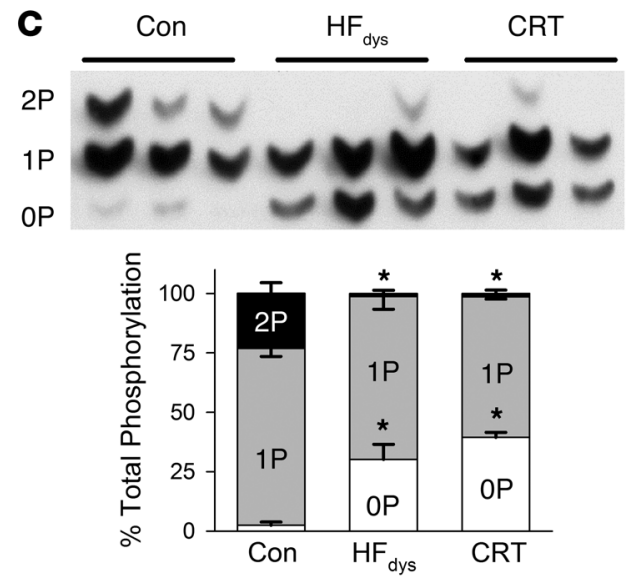

$\mathbf{E}$
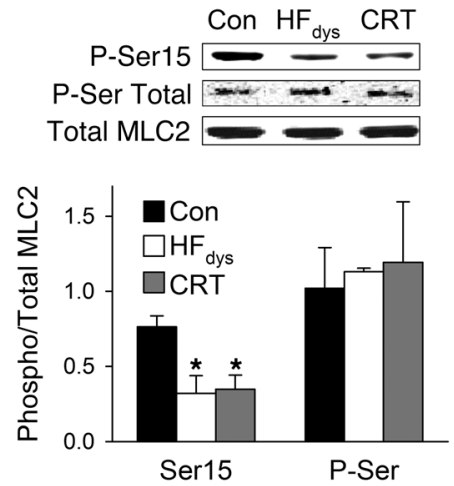
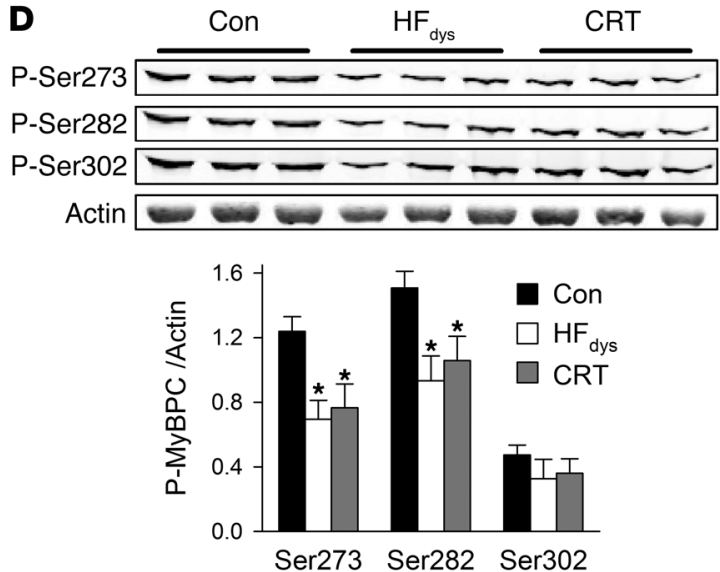

$\mathbf{F}$

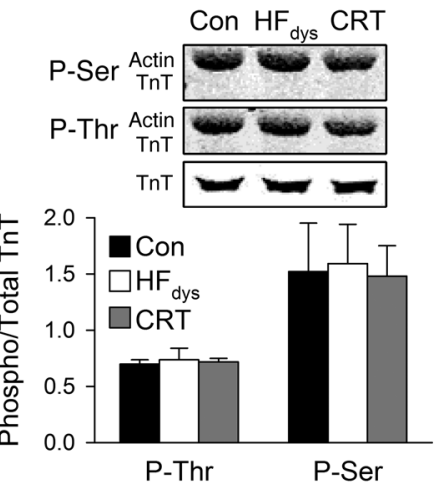

Figure 5

Calcium sensitization is mediated by phosphorylation, but not at the known targets. (A) Representative force-calcium curves for controls, $\mathrm{HF}_{\mathrm{dys}}$, and CRT before protein phosphatase-1 (PP1) and after treatment. (B) While there was no change in $\mathrm{F}_{\max }$, PP1 caused a desensitization in the controls $(n=6)$ and CRT $(n=5)$ groups, but had no effect on $\mathrm{HF}_{\text {dys }}(n=5)$. (C) Top: phos-Tag gel blotted for Tnl. Bottom: there was an increase in the unphosphorylated form and a decrease in the biphosphorylated form of Tnl in the HF dys and CRT groups $(n=3)$, indicating overall dephosphorylation of Tnl in HF. OP, unphosphorylated; 1P, phosphorylation state 1; 2P, phosphorylation state 2. (D) Top: Western blot with antibodies against 3 phosphorylation sites on MyBPC. Bottom: both $\mathrm{HF}_{\text {dys }}$ and CRT showed decreased phosphorylation at S273 and S282 $(n=3)$. (E) Top: Western blot against S15 phosphorylation on MLC2 and phospho-serine motif sites on MLC2. Bottom: $\mathrm{HF}_{\text {dys }}(n=4)$ and CRT $(n=4)$ were decreased from controls $(n=3)$, but no different from each other at S15, and there was no change in overall serine phosphorylation. (F) Top: phospho-motif antibodies for serine and threonine sites and total TnT. Bottom: there was very little phosphorylation of TnT in the 3 groups, with no differences among them $(n=3)$. ${ }^{*} P<0.05$ vs. control by 1 -way ANOVA.

contrast, myocytes from CRT hearts were unchanged by GSK$3 \beta$, consistent with existing stimulation of this pathway and underlying sensitization. Control myocytes were also unaffected by GSK- $3 \beta . \mathrm{F}_{\max }$ was unaltered by GSK-3 $\beta$ in any of the groups, agreeing with our finding that $\mathrm{F}_{\max }$ is primarily affected by myocyte geometry in these models.

To determine whether differential effects on calcium sensitivity were specific to GSK-3 $\beta$, we exposed $\mathrm{HF}_{\mathrm{dys}}$ and CRT myocytes to PKA and Akt, 2 kinases involved in GSK-3 $\beta$ signaling. As expected, PKA modestly increased $F_{\max }$ while decreasing calcium sensitivity (increased $\mathrm{EC}_{50}$ ), and it did so similarly in both groups (no interaction between group and treatment) (Supplemental Figure 22). This result is consistent with reduced TnI and MyBPC phosphorylation at PKA-targeted sites in both $\mathrm{HF}_{\text {dys }}$ and CRT (Figure 5, C and D). Although Akt is a recognized upstream kinase modulator of GSK-3 $\beta$, myocytes incubated with Akt exhibited no change in $\mathrm{F}_{\max }$ or $\mathrm{EC}_{50}$ in either group (Supplemental Figure 23, A and B), supporting an alternative pathway. While Akt treatment did not detectably increase phosphorylation of any proteins in the myofilament fraction (Supplemental Figure 23C), it did in whole-cell lysate (serine 9 
Table 1

Myofilament phosphorylation sites that are known to modulate calcium sensitivity

\begin{tabular}{|c|c|c|c|c|c|c|}
\hline Protein & $\begin{array}{l}\text { Phosphorylation } \\
\text { sites }\end{array}$ & Kinase & $\begin{array}{c}\text { Effect on } \\
\mathrm{Ca}^{2+} \text { sensitivity }\end{array}$ & $\begin{array}{l}\text { Phosphorylation } \\
\text { change in HF }\end{array}$ & $\begin{array}{c}\therefore \text { Effect on } \\
\text { sensitivity in HF }\end{array}$ & References \\
\hline Tnl & S22/S23 & PKA & $\downarrow$ & $\downarrow$ & $\uparrow$ & 29 \\
\hline Tnl & S43/S45 & PKC & $\downarrow$ & $\uparrow$ & $\downarrow$ & 30,31 \\
\hline Tnl & T144 & PKC & $\uparrow$ & $\uparrow$ & $\uparrow$ & 31,32 \\
\hline Tnl & S149/S150 & AMPK, PAK1 & $\uparrow$ & in vitro & $?$ & 33,34 \\
\hline MyBPC & S273/S282/S302 & PKA, others & $\uparrow$ & $\downarrow$ & $\downarrow$ & 35,36 \\
\hline TnT & T206 & PKC & $\downarrow$ & $?$ & $?$ & 38 \\
\hline TnT & S278/T287 & ROCKII & $\uparrow$ & $?$ & $?$ & 39 \\
\hline MLC2 & S15/S19 & MLCK & $\uparrow$ & $\downarrow$ & $\downarrow$ & 37 \\
\hline
\end{tabular}

The table provides the direction of a change in calcium sensitivity due to phosphorylation, the direction that phosphorylation is modified by HF, and how this in turn impacts calcium sensitivity.

on GSK-3 $\beta$; Supplemental Figure 23, D and E). These PKA and Akt data further support the specificity of the CRT myofilament response to GSK-3 $\beta$ phosphorylation of myofilament proteins.

Time course and offsetting effects on $\mathrm{Ca}^{2+}$ sensitivity by dyssynchrony. The observation that $\mathrm{Ca}^{2+}$ sensitivity declined with $\mathrm{HF}_{\text {dys }}$ but rose with $\mathrm{HF}_{\text {sync }}$ suggested differential phosphorylation might mediate dysfunction due to dyssynchrony. To test this further, we examined the time course of changes in both models, performing studies at 3 weeks in myocytes isolated from endocardial biopsies. After 3 weeks of pacing, both models showed a similar decline in $\mathrm{F}_{\max }$; however, there was dissociation of $\mathrm{Ca}^{2+}$ sensitivity (Figure 7, $\mathrm{A}$ and $\mathrm{B}$ ), with a fall in $\mathrm{EC}_{50}$ in $\mathrm{HF}_{\text {sync }}$ (sensitization), but no change in $\mathrm{HF}_{\text {dys }}$. There were no further declines in $\mathrm{HF}_{\text {sync }}$ at week 6, whereas $\mathrm{EC}_{50}$ rose in $\mathrm{HF}_{\text {dys. }}$ These data support an early sensitization factor in both models that is gradually offset by desensitization solely in $\mathrm{HF}_{\text {dys }}$ (Figure $7 \mathrm{C}$ ). This could relate to the decline in TnI phosphorylation by week 3 that remained similarly reduced at week 6 in both models (Figure 7, D and E). However, the ratio of phospho/total GSK-3 $\beta$ was unaltered at either time in $\mathrm{HF}_{\text {sync, }}$, whereas there was a gradual rise in the ratio in $\mathrm{HF}_{\text {dys }}$ (enzyme inactivation) concordant with the gradual increase in $\mathrm{EC}_{50}$. These results support the notion that phospho/total GSK-3 $\beta$ may serve as a biomarker for dyssynchrony with relevance to contractile function.

\section{Discussion}

We report that $\mathrm{HF}_{\mathrm{dys}}$ exhibits depressed myofilament responsiveness to calcium in both early and late activated regions and that CRT restores this to virtually normal levels. The mechanism appears to involve reactivation of GSK-3 $\beta$ and phosphorylation of Z-disk and M-band proteins as well as reversal of myocyte hypertrophy with depressed maximal force-per myocyte CSA. Similar myofilament functional improvement is attained if synchronous failing hearts are subjected to a period of dyssynchrony, confirming the key role of recoordinating contraction to this response. These results identify a mechanism by which CRT enhances systolic function beyond that previously attributed to chamber mechanics (3), adrenergic regulation $(19,20)$, and calcium homeostasis (17). The findings further suggest new approaches for improving myofilament function in the broader HF population.

Table 2

Myofilament phosphorylation sites increased by CRT

\begin{tabular}{|c|c|c|c|c|c|c|c|}
\hline Protein & Site & Loc Prob & Total Spectra & $P$ value & Peptide & Classification & Possible kinases \\
\hline Titin & S9901 & 1.00 & 9 & 0.010 & AVPPEEIPAMVVPPIPLLLPSPEEK & Myofilament & GSK3- $\beta$ \\
\hline Obscurin & S4809 & 0.81 & 45 & 0.042 & AGRPSTSPPASK & Myofilament/kinase & GSK3- $\beta$ \\
\hline abLIM1 & S421 & 0.96 & 50 & 0.044 & STSQGSISSPVYSR & Cytoskeleton/actin binding & p38, CDK5, GSK3- $\beta$ \\
\hline Tensin-1 & S1274 & 0.90 & 46 & 0.038 & $\begin{array}{l}\text { HPGAHPGGLASGVHGSAL- } \\
\text { GSPGSPSLGR }\end{array}$ & Focal adhesion/actin binding & p38, GSK3- $\beta$ \\
\hline THRAP-3 & S244 & 1.00 & 40 & 0.012 & ASAVSELsPR & Transcriptional coactivator & p38, GSK3- $\beta$ \\
\hline Nestin & S1062 & 1.00 & 15 & 0.031 & YLAEEETPEHMENPEsPR & Intermediate filament & p38, CDK5, GSK3- $\beta$ \\
\hline SORB-2 & S40 & 0.99 & 5 & 0.020 & DSQSPDSAKGFR & Z-Disk & p38, CDC2 \\
\hline SORB-2 & S231 & 1.00 & 44 & 0.004 & SHsDNGTDVFK & Z-Disk & CDC2, GSK3- $\beta$ \\
\hline SORB-2 & S307 & 1.00 & 34 & 0.035 & SSILQHERPPSLPTTPTPVSR & Z-Disk & PKA \\
\hline LMO-7 & S1346 & 1.00 & 114 & 0.020 & RGESLDNLDSPR & Cytoskeleton/actin binding & PKA \\
\hline LDB3/cypher & S507 & 1.00 & 110 & 0.014 & GAPTYGPPGPQVsPLAR & Z-Disk & CDK5 \\
\hline SPEG & S2039 & 1.00 & 28 & 0.039 & AAsVELPQR & Kinase & PKC \\
\hline Filamin-C & S2228 & 1.00 & 109 & 0.011 & LGsFGSITR & Z-Disk/actin binding & PKB \\
\hline Myotilin 1 & $\mathrm{~S} 231$ & 0.90 & 20 & 0.020 & SSSRGDVNDQDAIQEK & Z-Disk & PKG, PKA \\
\hline Leiomodin-2 & S513 & 0.98 & 8 & 0.039 & GTPGSSPYAsPR & Binds tropomyosin & CDK5, p38 \\
\hline
\end{tabular}

The peptide sequence is shown, with the phosphorylated site indicated in lowercase. Possible kinases were determined using the Human Protein Reference Database and NetphosK. Loc prob, Localization probability as determined by the A-Score algorithm using scaffold PTM; THRAP-3, thyroid hormone receptor-associated protein 3; SORB-2, sorbin and SH3 domain-containing protein 2; LDB3, LIM domain-binding protein 3; LMO7, LIM domain only protein 7. 
A

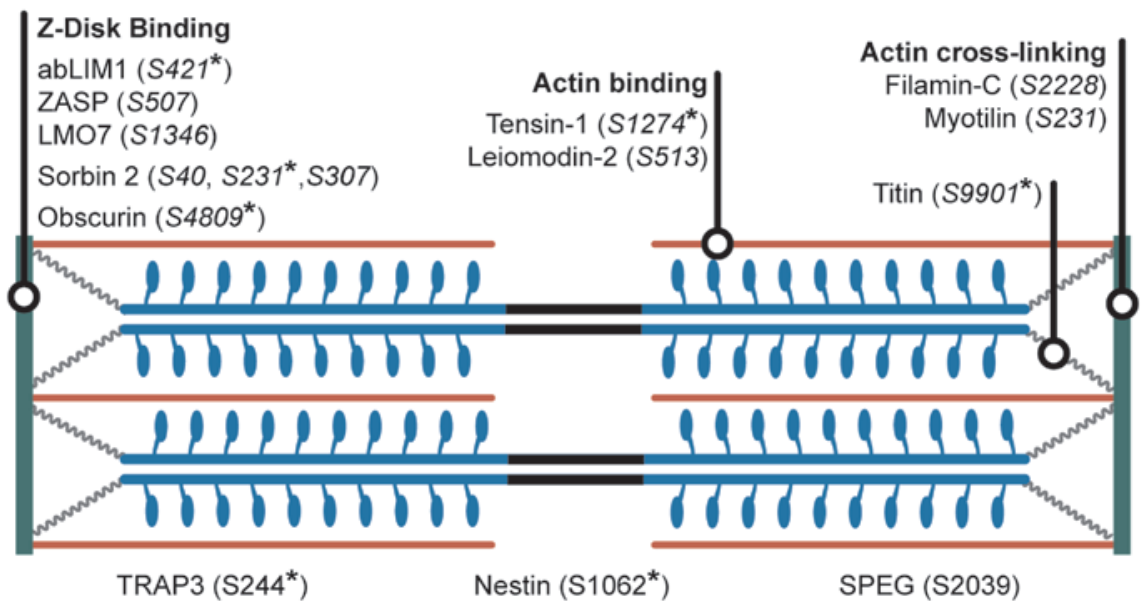

B
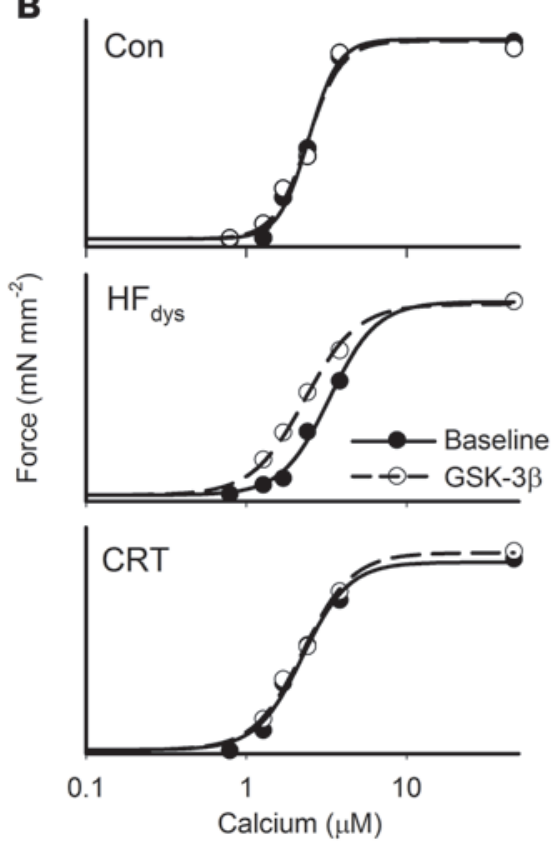

C

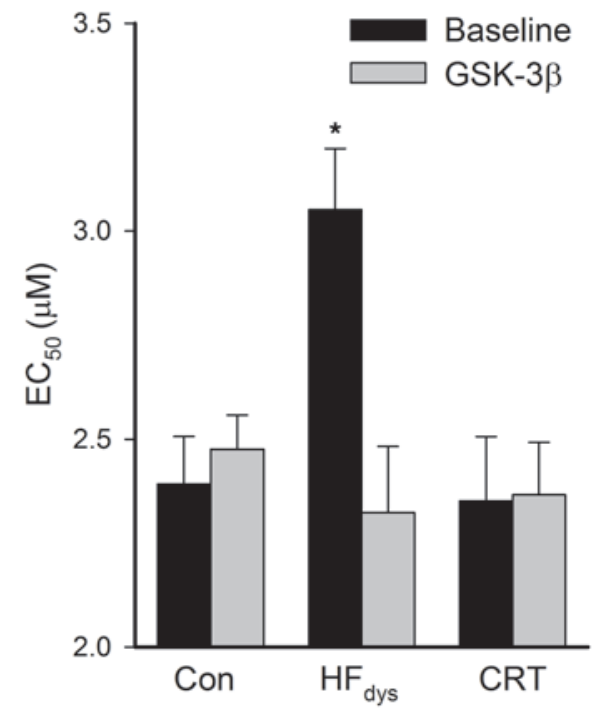

Figure 6

Increased phosphorylation at GSK-3 $\beta$ sites in CRT mediated the calcium-sensitizing effect. (A) Schematic of the cardiac sarcomere showing Z-disk (green), titin (gray) thick filament (blue), M-line (black), and thin filament (red). The proteins (and sites) that were identified as increased with CRT are shown, with their best-known localization. Asterisks indicate matches with GSK-3 $\beta$ consensus sequence. (B) Representative force-calcium relationships in controls (top), $\mathrm{HF}_{\text {dys }}$ (middle), and CRT (bottom) before (solid lines) and after GSK-3 $\beta$ treatment (dashed lines). (C) There was a calcium-sensitizing effect of GSK-3 $\beta$ in $\mathrm{HF}_{\text {dys }}(n=8)$, bringing it to the same level as controls and CRT. GSK- $3 \beta$ had no effect on controls $(n=5)$ or CRT myocytes $(n=8) .{ }^{*} P<0.05$ vs. control baseline value.
Dyssynchrony generates regional heterogeneity of wall stress in the ventricle, with areas of increased or decreased load corresponding, respectively, to late or early activation. Despite these differences, similar depression of myofilament function and its recovery by CRT were observed in both regions. This indicates that the precise pattern of stress (or strain) is less important than the presence of spatial heterogeneity of these properties. Global chamber modifications with both dyssynchronous and resynchronized HF are not unique to myofilament function, but have also been observed with respect to antiapoptosis signaling coupled to Akt and GSK-3 $\beta$ (11), ion channel function (17), and adrenergic regulation (19).

Successful CRT converts hearts with a prior history of dyssynchrony into those with coordinate contraction. This temporal sequence was central to enhancing myofibrillar function just as it was to improving myocardial responsiveness to $\beta$-adrenergic stimulation(19). Failing hearts that were never dyssynchronous $\left(\mathrm{HF}_{\text {sync }}\right)$ had worse myofilament function than those that were resynchronized, the latter including CRT, V3A3, and AVA models. The presence of dyssynchrony differentially affected calcium sensitivity.
Enhanced $\mathrm{Ca}^{2+}$ sensitivity as observed with $\mathrm{HF}_{\text {sync }}$ has been reported in human $\mathrm{HF}_{\text {sync }}(40)$, whereas reduced $\mathrm{Ca}^{2+}$ sensitivity as with $\mathrm{HF}_{\mathrm{dys}}$ was compatible with experimental data from failing hearts with myocardial infarction (24). These differences were previously attributed to difficulties in preserving the phosphorylation state of myofilament proteins in human explant tissue samples (41). However, the current data controls for this (dogs do not receive different medications and tissue is harvested almost immediately after sacrifice), suggesting instead that regional stress-strain heterogeneity, as occurs with electromechanical dyssynchrony or regional infarction, plays a role. Disparities in $\mathrm{Ca}^{2+}$ sensitivity between $\mathrm{HF}_{\text {sync }}$ and $\mathrm{HF}_{\text {dys }}$ may underlie the greater adverse risks (increased morbidity and mortality) observed in HF patients with dyssynchrony $(42,43)$.

The AVA study that exposed hearts to temporary dyssynchrony also increased $\mathrm{F}_{\max }$ over $\mathrm{HF}_{\text {sync }}$ and suggests what we believe is a novel translational approach for improving myofibrillar function in the failing heart. For example, patients with $\mathrm{HF}_{\text {sync }}$ might benefit from a defined exposure to RV pacing-induced dyssynchrony that is then terminated to activate ameliorative changes. Alter- 
Table 3

Phosphorylation sites increased by CRT that were also in vitro targets of GSK-3 $\beta$

\begin{tabular}{|c|c|c|c|c|c|}
\hline Protein & Site & Peptide & $\mathrm{HF}_{\mathrm{dys}}$ & $\mathrm{HF}_{\mathrm{dys}}+\mathrm{GSK}-3 \beta$ & Predicted target \\
\hline Obscurin & S4809A & AGRPSTSPPASK & Labeled & Labeled & $\mathrm{x}$ \\
\hline abLIM1 & $\mathrm{S} 421^{\mathrm{A}}$ & STSQGSISSPVYSR & Labeled & Labeled & $X$ \\
\hline Tensin-1 & S1274 & HPGAHPGGLASGVHGSALGSPGsPSLGR & & Present & $\mathrm{X}$ \\
\hline THRAP-3 & S244 & ASAVSELsPR & Present & Present & $\mathrm{X}$ \\
\hline LM07 & S1346 & RGEsLDNLDSPR & Labeled & Labeled & \\
\hline LDB3/Cypher & S507 & GAPTYGPPGPQVsPLAR & Present & Present & \\
\hline SPEG & S2039 & AAsVELPQR & & Present & \\
\hline Filamin-C & S2228 & LGsFGSITR & Present & Labeled & \\
\hline
\end{tabular}

Labeled sites incorporated the ${ }^{18} \mathrm{O}$-labeled ATP. Predicted target indicates whether the phosphorylation site was identified as a possible substrate for GSK-3 $\beta$ using in silico analysis (see Table 2). For each group, $n=3$ dogs were pooled. ALow localization probability of the phospho-serine, indicating a nearby serine could also be a possible target.

natively, patients receiving CRT might benefit from periods of suspending biventricular pacing. This phenomenon may reflect a form of dyssynchrony-induced "preconditioning" (44), establishing molecular signaling, such as GSK-3 $\beta$ inactivation, that triggers subsequent changes after hearts are subsequently resynchronized. The precise molecular pathways involved, the optimal duration of dyssynchrony exposure needed to achieve them, and the longevity of any ameliorative myofilament effects upon resynchronization remain to be fully determined.

$\mathrm{F}_{\max }$ declined with $\mathrm{HF}_{\text {dys }}$ and was restored by CRT. Neither myofibrillar protein degradation nor isoform shifts were detected, but $\mathrm{HF}_{\text {dys }}$ myocytes were larger than controls. Myocyte hypertrophy with dyssynchrony could result from heightened regional stresses (systolic and diastolic) that occur in early- and late-contracting regions. $\mathrm{HF}_{\text {dys }}$ amplifies p38 and CaMKII activity that can stimulate hypertrophy (45), and CRT restores both to normal (11). Cell hypertrophy is expected to elevate force due to more myofibers, yet maximal force was unchanged, implying force per fiber declined in $\mathrm{HF}_{\text {dys }}$ and was restored to normal by CRT. Similar results have appeared in several prior studies, though they were not specifically highlighted. For example, in HF patients treated with LV assist devices (LVAD), $\mathrm{F}_{\max }$ (normalized to cell area) was one-fourth that of healthy control levels prior to LVAD insertion and doubled after LVAD insertion (46). However, myocyte size was 4 times greater than normal before LVAD insertion and fell by $50 \%$ after LVAD insertion (cells got smaller). Multiplying normalized $\mathrm{F}_{\max }$ by cell size in each case yielded the same maximal force value in all 3 groups. Another example comes from a study of myocardial infarction: normalized $F_{\max }$ was lower in the infarcted samples (compared with controls), but cell size was larger. When $\mathrm{F}_{\max }$ was not adjusted for cell area (unnormalized), it was similar between the groups (24). Similar findings were reported in myocytes from RV hypertrophy (47). There are multiple possible explanations for unchanged nonnormalized $F_{\max }$ with myocyte hypertrophy, including (a) myofibrils are not added despite an increase in cell size, (b) new myofibrils are added, but they function poorly, or (c) overall per-myofiber function is depressed. Which of these possibilities is responsible, however, remains unknown.

Modulation of $\mathrm{Ca}^{2+}$-sensitivity $\left(\mathrm{EC}_{50}\right)$ by phosphorylation, as was found relevant to CRT, commonly involves the thin-filament proteins. Modifications in TnI, MyBPC, and MCL2 were all documented in the present study, but were similar in both $\mathrm{HF}_{\text {dys }}$ and CRT. CRT also depresses TnI phosphorylation at S165, T180, and S198 (48), but these all go in the opposite direction to explain a PP1 effect. Rather than revealing alternative thin-filament targets, our proteomic analysis identified Z-disk and/or M-band proteins. Both of these regions are nexuses for mechanosensing, and while their possible involvement with contractile-protein regulation is less well characterized, they anchor thin and thick filaments, respectively, and localize enzymes that modify myofilament proteins. They are central to maintaining sarcomere geometry, integrating force, and transmitting tension, so it is reasonable that they could also modulate $\mathrm{Ca}^{2+}$-dependent contraction. For example, modifying the Z-disk proteins Cap-Z (reducing expression) or muscle LIM protein (MLP) (acetylation) increases calcium sensitivity $(49,50)$. In skeletal muscle, reducing Z-disk or M-band stiffness reduces calcium sensitivity (51), while genetic mutations in Z-disk proteins result in cardiomyopathy associated with Z-disk destabilization (52) and/or changes in myofilament stiffness and $\mathrm{Ca}^{2+}$ response (53).

Treating $\mathrm{HF}_{\text {dys }}$ myofilament samples with active GSK-3 $\beta$ recapitulated both the sensitization of $\mathrm{HF}_{\text {dys }}$ myofibers and key features of the CRT phospho-proteomic footprint (Table 2 and Table 3), including sites that were not predicted by bioinformatics. GSK-3 $\beta$ has been previously only shown to modify MyBPC and thereby affect contraction kinetics (54). The current data are the first, to our knowledge, to reveal its effects on calcium sensitivity, but this was unique to myocytes from HF with dyssynchronous contraction. GSK-3 $\beta$ phosphorylation was not due to Akt, as the latter kinase had no effect on myofibrillar phosphorylation or function despite its capacity to phosphorylate cytosolic GSK-3 $\beta$.

Among the various myofilament GSK-3 $\beta$ targets, the more intriguing include tensin-1 (S1274) and filamin-C (S2228), as these modifications were not detected in the absence of kinase incubation. Tensin-1, classically known as a focal adhesion protein, binds to the $\mathrm{Z}$-disk in cardiomyocytes in response to stress (55). Filamin-C is an actin-binding protein, localized within the Z-disk. Mutations in filamin-C cause dilated cardiomyopathy (DCM), and a splice variant is associated with heart disease (56). Other potential candidates include obscurin (S4809) and actinbinding LIM protein 1 (abLIM1) (S421). Obscurin is present in the Z-disk and M-band, where it is tethered to titin and myomesin, respectively, and it contains a kinase domain as well. abLIM1 binds to actin at the $\mathrm{Z}$-disk, and the phosphorylation site that was altered by CRT (S421) is in the region thought to bind to actin (57). None of these proteins or their phosphorylated forms have been previously associated with changes in myofilament function under normal or pathological conditions. 


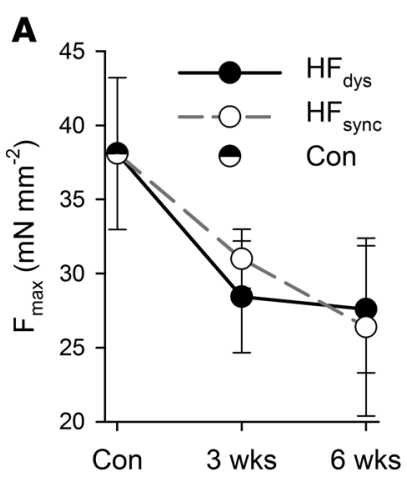

D

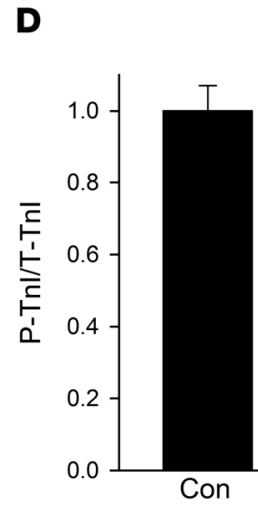

B

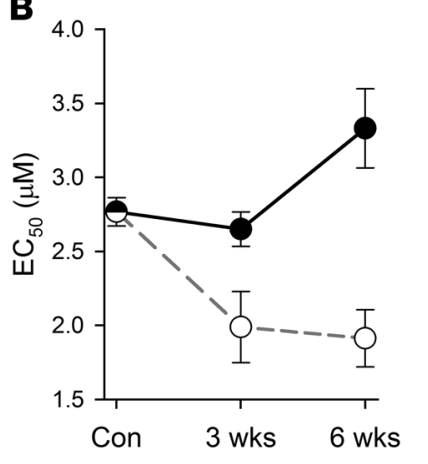

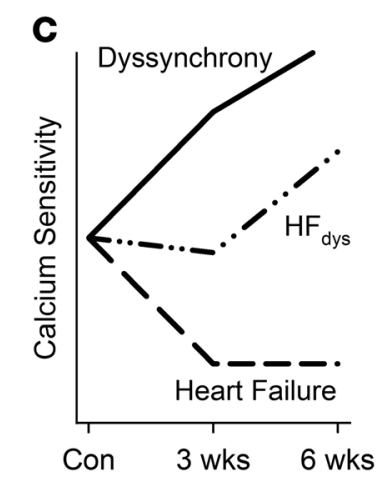

E

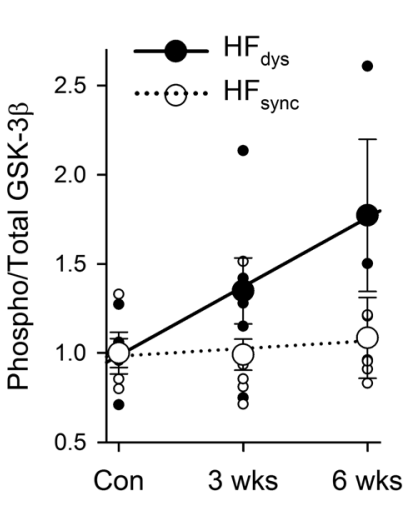

\section{Figure 7}

Time course of function and phosphorylation during dyssynchrony and HF. (A) Endocardial biopsies taken at the 3-week time point (controls, $n=8$, $\mathrm{HF}_{\text {dys }} 3$ weeks: $n=11, \mathrm{HF}_{\text {sync }} 3$ weeks: $n=5, \mathrm{HF}_{\text {dys }} 6$ weeks: $n=17, \mathrm{HF}_{\text {sync }} 6$ weeks: $n=18$ myocytes) showed that $\mathrm{F}_{\text {max }}$ decreased steadily over the entire 6-week protocol. (B) Calcium sensitivity was altered in $\mathrm{HF}_{\text {sync }}$ by 3 weeks, with no changes between 3 and 6 weeks. However, $\mathrm{HF}_{\text {dys }}$ was unaltered at 3 weeks, but desensitized between 3 and 6 weeks. (C) When the sensitizing temporal effect of decreased Tnl phosphorylation is added to the desensitizing temporal effects of decreased GSK-3 $\beta$ activity, it recapitulates the observed phenotype in B. (D) S22/S23 Tnl phosphorylation was decreased in both $\mathrm{HF}_{\text {dys }}$ and $\mathrm{HF}_{\text {sync }}$ by 3 weeks, with no additional decrease between 3 and 6 weeks ( $n=5$ per time point). (E) Phospho GSK-3 $\beta$ was unchanged in $\mathrm{HF}_{\text {sync }}$ over the 6 weeks, but linearly increased in $\mathrm{HF}_{\text {dys }} ; P<0.01$ via multivariate regression analysis. ${ }^{*} P<0.05$ vs. controls; $\# P<0.05$ vs. $\mathrm{HF}_{\text {sync }}$ at 3 weeks.

Our study leaves unanswered the exact identity of which GSK-3 $\beta$ target or targets are responsible for improved calcium sensitivity by CRT. The M-band and Z-disk are highly structured regions in the sarcomere, and protein substitution cannot be achieved by diffusion in skinned cell preparations. Thus, direct confirmation will require site mutagenesis and genetic modifications. Ongoing efforts to translate the canine model to the mouse will be an important next step to dissecting the pathways, since gene delivery and/or transgenic models are more practical. This will also allow genetic knockdown of GSK-3 $\beta$ to confirm the myofilament substrates. The canine model is highly translational but poses its own limitations, since tachypacing is required throughout the protocol to generate HF. This impacts global functional parameters such as stroke volume or ejection fraction, which decline at fast heart rates independently of contractility changes (58). This likely explains why such variables and chamber volumes were similar among the models despite differences in myofilament contractile function, whereas the less load-sensitive parameter $\mathrm{dP} / \mathrm{dt}_{\max } / \mathrm{IP}$ (IP, instantaneous developed pressure at time of $\mathrm{dP} / \mathrm{dt}_{\max }$ ) was improved by CRT.

The myofilament is rapidly becoming an attractive target for enhancing systolic function in $\mathrm{HF}(21)$. Over the past 10 to 20 years, investigators have explored drugs to improve $\mathrm{Ca}^{2+}$ responsiveness, though such efforts have yet to yield a broadly successful therapy. A new approach to enhancing myofilament ATPase activity was reported (21) and is currently in clinical trials (23), though chronic efficacy remains unknown. In contrast, CRT is already a successful treatment with documented long-term benefits on morbidity and mortality. The present analysis attempts to reverse engineer it, unmasking an unanticipated yet striking improvement in myofilament function by a mechanism likely to be both safe and effective. At present, we do not know whether there is a single Z-disk or M-band protein target or whether several are involved, but the results strongly suggest GSK-3 $\beta$ as a key signaling kinase, revealing what we believe to be a novel role for this enzyme in the sarcomere. The ability to mimic $\mathrm{Ca}^{2+}$-sensitizing effects of CRT by augmenting GSK-3 $\beta$ activation in $\mathrm{HF}_{\mathrm{dys}}$ myocytes and/or exposing the heart to brief periods of sustained dyssynchrony may help forge new paths for HF treatment.

\section{Methods}

Canine pacing models. Details of the canine models have been previously reported $(19,20)$. All dogs except controls received a pacemaker implant (Medtronic). $\mathrm{HF}_{\mathrm{dys}}$ and CRT models both received left bundle branch radio-frequency ablation at time of pacemaker implant, while in 3 other models ( $\mathrm{HF}_{\text {sync }}$ AVA, 
V3A3), the left bundle remained intact. The location and timing of pacing varied among the experimental models and are detailed in Figure 1. All dogs were provided 7 to 10 days for full recovery prior to initiating 6 weeks of tachypacing $(200 \mathrm{bpm})$. After 3 weeks of pacing, a subset of dogs was placed under anesthesia and a 7F bioptome (Cordis Corp.) was used to collect 5-7 biopsies from the endocardium near the RV apex under fluoroscopic guidance.

At terminal study, animals were anesthetized with pentobarbital, hearts excised under ice-cold cardioplegia, and tissue prepared for analysis. Supplemental Table 1 provides global hemodynamics and echocardiographic data for each model - revealing the presence of cardiac failure at the chamber level compared with normal controls. All models display chamber dilation, systolic, and diastolic dysfunction, though the CRT model had improved systolic function $\left(\mathrm{dP} / \mathrm{dt}_{\max } / \mathrm{IP}\right)$. Other details are provided in Supplemental Methods and Supplemental Table 1.

Myofilament function. Right heart trabeculae were isolated and skinned overnight in the presence of $1 \%$ Triton X-100 and protease (Sigma-Aldrich) and phosphatase inhibitors (Roche Diagnostics). Trabeculae were attached to a force transducer (SI Heidelberg) and a stationary hook. Force was measured as bath $\mathrm{Ca}^{2+}$ concentration was increased from 0 to saturating conditions. In a subset of studies, trabeculae were subsequently treated with the phosphatase PP1 $(25,000 \mathrm{mU} / \mathrm{ml} \times 60$ minutes; New England Biolabs $)$ and a second force-calcium relation determined.

As trabeculae could not be isolated from the LV free wall, studies were also performed in isolated myocytes. Companion studies from the LV septum were also conducted. For myocyte analysis, flash-frozen endomyocardium was homogenized in Triton X-100 and protease and phosphatase inhibitors and the mixture disaggregated by low-speed pulverization to generate a skinned myocyte preparation. Myocytes were then attached to a force transducer and motor arm. Once attached, the protocol was similar to that used in trabeculae. Subsets of myocytes were exposed to either

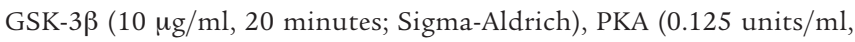
20 minutes; Sigma-Aldrich), or Akt (10 $\mu \mathrm{g} / \mathrm{ml}, 20$ minutes; Sigma-Aldrich). Force-calcium data were collected before and after treatment.

Force-calcium data were fit to the Hill equation: $\mathrm{F}=\mathrm{F}_{\max } \times \mathrm{Ca}^{\mathrm{h}} /\left(\mathrm{EC}_{50}^{\mathrm{h}}+\mathrm{Ca}^{\mathrm{h}}\right)$, yielding $\mathrm{F}_{\max }$, calcium sensitivity $\left(\mathrm{Ca}^{2+}\right.$ required to achieve $50 \%$ maximal force; $\mathrm{EC}_{50}$ ), and cooperativity (Hill coefficient, $h$ ).

Phos-Tag and Western blots. Tissue samples from control, $\mathrm{HF}_{\mathrm{dys}}$, and CRT hearts were processed in the presence of protease and phosphatase inhibitors to obtain a myofilament-enriched protein sample (Supplemental Methods). Samples were run on phos-Tag gels (Wako Pure Chemical Industries) and transferred to a membrane; cardiac TnI was detected with the 8I-7 TnI antibody (Spectral Diagnostics). For other proteins, immunoblots were performed using MyBP-C antibodies against phospho-S273, phosphoS282, phospho-S302, and total (gift from Sakthivel Sadayappan, Loyola University, Chicago, Illinois, USA), MLC-2 phospho-antibodies against phospho-S15 (gift from Neal Epstein, NIH, Bethesda, Maryland, USA), phospho-TnI (Cell Signaling), total TnI (Spectral Diagnostics), phosphoserine motif (Zymed Laboratories), phospho-threonine motif (Zymed), TnT (Cell Signaling), total MLC2 (Novus Biologicals), P-S9 GSK-3 $\beta$ (Sigma-Aldrich), and/or total GSK-3 $\beta$ (Santa Cruz Biotechnology Inc.). Blots were scanned using an Odyssey Infrared Imager (Li-Cor Biosciences) and analyzed using Odyssey Application software (v3.0.30, Li-Cor Biosciences). Several of these antibodies had no prior publications supporting their use in canine heart tissue. In these instances, antibodies were optimized and verified by comparing Western blots performed in alternate species (mouse). Fortunately, the myofilament proteins share very high amino acid homology across mammalian species.
Mass spectrometry. Six $\mathrm{HF}_{\mathrm{dys}}$ and $6 \mathrm{CRT} \mathrm{LV}$ lateral wall myocardial samples were myofilament enriched ( $1 \mathrm{mg}$ protein in $8 \mathrm{M}$ urea, $1 \% \mathrm{SDS}$ ) and digested in trypsin (Promega Corp.). Alternatively, myofilament-enriched samples from $\mathrm{HF}_{\text {dys }}$ tissue were split into 2 equal parts, one incubated in ATP $\gamma$ - $\mathrm{P}^{18} \mathrm{O}$-labeled (Cambridge Isotope Laboratories Inc.) relax buffer, and the second incubated in labeled relax buffer plus $4 \mu \mathrm{g}$ of GSK-3 $\beta(n=3)$. The labeled relax buffer contained a 1:1 ratio of unlabeled and ${ }^{18} \mathrm{O}$-labeled ATP. The samples were incubated at $30^{\circ} \mathrm{C}$ for 30 minutes.

All myofilament samples were fractionated by strong-cation exchange to decrease sample complexity and increase sequence coverage. Each fraction was then individually phospho enriched using titanium oxide affinity chromatography. Phospho-peptides were analyzed on an Agilent 1200 Nano-LC system (Agilent Technologies) connected to an LTQOrbitrap mass spectrometer (Thermo Scientific) equipped with a nanoelectrospray ion source.

All raw MS/MS data was searched using the Sorcerer 2-SEQUEST algorithm (Sage-N Research). Additional analysis was done using Scaffold PTM (v1.1.3; Proteome Software). For studies with GSK-3 $\beta$ treatment, MS data were searched using SEQUEST, with labeled phosphorylation that included an additional 6.01-Da shift due to the ${ }^{18} \mathrm{O}$-labeled phosphate group. Kinase consensus amino acid sequences were predicted using NetPhosK (http://cbs.dtu.dk/services/NetPhosK/) and the Human Protein Reference Database (hprd.org).

Further details are provided in the Supplemental Methods.

Statistics. Values were compared between the groups using 1-way ANOVA, with post hoc comparisons made with the Holm-Sidak method using SigmaPlot (v11, Systat Software Inc.). MS was quantified based on label-free spectra counts. Normalized spectral counts were compared using Student's $t$ test. GSK-3 $\beta$ phosphorylation relationships were tested using multivariate regression analysis using Systat (Systat Software, Inc.). All tests were 2 -tailed with a $P$ value of less than 0.05 considered significant. All values are presented as mean \pm SEM.

Study approval. All animal studies were approved by the Johns Hopkins Animal Care and Use Committee and performed by trained personnel.

\section{Acknowledgments}

This work was supported by the NIH grants P01-HL077180 (to D.A. Kass and J. Van Eyk), HL62426 (to P.P. de Tombe), HL75494 (to P.P. de Tombe), and T32-HL007227 (to J. Van Eyk and D.A. Kass); a Fondation Leducq grant (to D.A. Kass); a Peter Belfer Laboratory grant (to D.A. Kass), Proteomic Initiative contracts NHLBI-HV-10-05(2) (to J. Van Eyk) and HHSN268201000032C (to J. Van Eyk and D.A. Kass); the Abraham and Virginia Weiss Professorship (to D.A. Kass); and an American Heart Association post-doctoral fellowship (to J.A. Kirk, 11POST7210031). We thank Sakthivel Sadayappan and Neal Epstein for the use of their custom antibodies. Publication of this article was funded in part by the Open Access Promotion Fund of the Johns Hopkins University Libraries.

Received for publication February 8, 2013, and accepted in revised form September 19, 2013.

Address correspondence to: David A. Kass, Division of Cardiology, Johns Hopkins Medical Institutions, Ross Research Building, Room 858, 720 Rutland Avenue, Baltimore, Maryland 21205, USA. Phone: 410.955.7153; Fax: 410.502.2558; E-mail: dkass@jhmi.edu.
1. Metra M, Bettari L, Carubelli V, Cas LD. Old and new intravenous inotropic agents in the treatment of advanced heart failure. Prog Cardiovasc Dis. 2011;
54(2):97-106

2. Landmesser U, Drexler H. Update on inotropic therapy in the management of acute heart failure. Curr
Treat Options Cardiovasc Med. 2007;9(6):443-449.

3. Kass DA, et al. Improved left ventricular mechanics from acute VDD pacing in patients with dilated 
cardiomyopathy and ventricular conduction delay. Circulation. 1999;99(12):1567-1573.

4. Nelson GS, et al. Left ventricular or biventricular pacing improves cardiac function at diminished energy cost in patients with dilated cardiomyopathy and left bundle-branch block. Circulation. 2000; 102(25):3053-3059.

5. Ukkonen H, et al. Effect of cardiac resynchronization on myocardial efficiency and regional oxidative metabolism. Circulation. 2003;107(1):28-31.

6. Cleland JG, et al. The effect of cardiac resynchronization on morbidity and mortality in heart failure. N Engl J Med. 2005;352(15):1539-1549.

7. Bristow MR, et al. Cardiac-resynchronization therapy with or without an implantable defibrillator in advanced chronic heart failure. $N$ Engl J Med. 2004; 350(21):2140-2150.

8. Chalil S, et al. Intraventricular dyssynchrony predicts mortality and morbidity after cardiac resynchronization therapy: a study using cardiovascular magnetic resonance tissue synchronization imaging. J Am Coll Cardiol. 2007;50(3):243-252.

9. Gorcsan J 3rd, et al. Relationship of echocardiographic dyssynchrony to long-term survival after cardiac resynchronization therapy. Circulation. 2010; 122(19):1910-1918.

10. Barth AS, et al. Cardiac resynchronization therapy corrects dyssynchrony-induced regional gene expression changes on a genomic level. Circ Cardiovasc Genet. 2009;2(4):371-378.

11. Chakir K, et al. Reversal of global apoptosis and regional stress kinase activation by cardiac resynchronization. Circulation. 2008;117(11):1369-1377.

12. D’Ascia C, Cittadini A, Monti MG, Riccio G, Saccà L. Effects of biventricular pacing on interstitial remodelling, tumor necrosis factor-alpha expression, and apoptotic death in failing human myocardium. Eur Heart J. 2006;27(2):201-206.

13. Nishijima Y, et al. Chronic cardiac resynchronization therapy and reverse ventricular remodeling in a model of nonischemic cardiomyopathy. Life Sci. 2007;81(14):1152-1159.

14. Mullens W, et al. Early and late effects of cardiac resynchronization therapy on force-frequency relation and contractility regulating gene expression in heart failure patients. Heart Rhythm. 2008;5(1):52-59.

15. Sachse FB, et al. Subcellular structures and function of myocytes impaired during heart failure are restored by cardiac resynchronization therapy. Circ Res. 2012;110(4):588-597.

16. Wang SB, Foster DB, Rucker J, O'Rourke B, Kass DA, Van Eyk JE. Redox regulation of mitochondrial ATP synthase: implications for cardiac resynchronization therapy. Circ Res. 2011;109(7):750-757.

17. Aiba T, et al. Electrophysioloigcal consequences of dyssynchronous heart failure and its restoration by resynchronization therapy. Circulation. 2009; 119(9):1220-1230.

18. Aiba T, Tomaselli G. Electrical remodeling in dyssynchrony and resynchronization. $J$ Cardiovasc Transl Re. 2012;5(2):170-179.

19. Chakir K, et al. Galphas-biased $\beta 2$-adrenergic receptor signaling from restoring synchronous contraction in the failing heart. Sci Transl Med. 2011; 3(100):100ra188.

20. Chakir K, et al. Mechanisms of enhanced betaadrenergic reserve from cardiac resynchronization therapy. Circulation. 2009;119(9):1231-1240.

21. Malik FI, et al. Cardiac myosin activation: a potential therapeutic approach for systolic heart failure. Science. 2011;331(6023):1439-1443.

22. Sorsa T, Pollesello P, Solaro RJ. The contractile apparatus as a target for drugs against heart failure: interaction of levosimendan, a calcium sensitiser, with cardiac troponin c. Mol Cell Biochem. 2004; 266(1-2):87-107.

23. Cleland JG, et al. The effects of the cardiac myosin activator, omecamtiv mecarbil, on cardiac function in systolic heart failure: a double-blind, placebocontrolled, crossover, dose-ranging phase 2 trial. Lancet. 2011;378(9792):676-683.

24. Belin RJ, et al. Augmented protein kinase C-alphainduced myofilament protein phosphorylation contributes to myofilament dysfunction in experimental congestive heart failure. Circ Res. 2007; 101(2):195-204.

25. Kooij V, et al. Protein kinase C alpha and epsilon phosphorylation of troponin and myosin binding protein $\mathrm{C}$ reduce $\mathrm{Ca} 2+$ sensitivity in human myocardium. Basic Res Cardiol. 2010;105(2):289-300.

26. Solaro RJ, van der Velden J. Why does troponin I have so many phosphorylation sites? Fact and fancy. J Mol Cell Cardiol. 2010;48(5):810-816.

27. Murphy AM, et al. Transgenic mouse model of stunned myocardium. Science. 2000; 287(5452):488-491.

28. Nassar R, et al. cTnT1, a cardiac troponin T isoform, decreases myofilament tension and affects the left ventricular pressure waveform. Am J Physiol Heart Circ Physiol. 2005;288(3):H1147-H1156.

29. Pi Y, Zhang D, Kemnitz KR, Wang H, Walker JW. Protein kinase $\mathrm{C}$ and $\mathrm{A}$ sites on troponin I regulate myofilament $\mathrm{Ca}^{2+}$ sensitivity and ATPase activity in the mouse myocardium. J Physiol. 2003; 552 (pt 3):845-857.

30. Burkart EM, et al. Phosphorylation or glutamic acid substitution at protein kinase $\mathrm{C}$ sites on cardiac troponin I differentially depress myofilament tension and shortening velocity. J Biol Chem. 2003; 278(13):11265-11272.

31. Kirk JA, et al. Left ventricular and myocardial function in mice expressing constitutively pseudophosphorylated cardiac troponin I. Circ Res. 2009; 105(12):1232-1239.

32. Wang H, Grant JE, Doede CM, Sadayappan S, Robbins J, Walker JW. PKC- $\beta$ II sensitizes cardiac myofilaments to $\mathrm{Ca}^{2+}$ by phosphorylating troponin I on threonine-144. J Mol Cell Cardiol. 2006;41(5):823-833.

33. Nixon BR, et al. AMP-activated protein kinase phosphorylates cardiac troponin I at Ser-150 to increase myofilament calcium sensitivity and blunt PKA-dependent function. J Biol Chem. 2012; 287(23):19136-19147.

34. Buscemi N, Foster DB, Neverova I, Van Eyk JE. p21-activated kinase increases the calcium sensitivity of rat triton-skinned cardiac muscle fiber bundles via a mechanism potentially involving novel phosphorylation of troponin I. Circ Res. 2002; 91(6):509-516.

35. Chen PP, Patel JR, Rybakova IN, Walker JW, Moss RL. Protein kinase A-induced myofilament desensitization to $\mathrm{Ca}(2+)$ as a result of phosphorylation of cardiac myosin-binding protein C. J Gen Physiol. 2010;136(6):615-627.

36. Sadayappan S, et al. A critical function for Ser282 in cardiac Myosin binding protein-C phosphorylation and cardiac function. Circ Res. 2011; 109(2):141-150.

37. van der Velden $\mathrm{J}$, et al. The effect of myosin light chain 2 dephosphorylation on $\mathrm{Ca} 2+$-sensitivity of force is enhanced in failing human hearts. Cardiovasc Res. 2003;57(2):505-514.

38. Sumandea MP, Pyle WG, Kobayashi T, de Tombe $\mathrm{PP}$, Solaro RJ. Identification of a functionally critical protein kinase $\mathrm{C}$ phosphorylation residue of cardiac troponin T. J Biol Chem. 2003; 278(37):35135-35144.

39. Vahebi S, Kobayashi T, Warren CM, de Tombe PP Solaro RJ. Functional effects of rho-kinase-dependent phosphorylation of specific sites on cardiac troponin. Circ Res. 2005;96(7):740-747.

40. Wolff MR, Buck SH, Stoker SW, Greaser ML, Mentzer RM. Myofibrillar calcium sensitivity of isometric tension is increased in human dilated car- diomyopathies: role of altered beta-adrenergically mediated protein phosphorylation. J Clin Invest. 1996;98(1):167-176.

41. Marston SB, de Tombe PP. Troponin phosphorylation and myofilament $\mathrm{Ca} 2+$-sensitivity in heart failure: increased or decreased? J Mol Cell Cardiol. 2008; 45(5):603-607.

42. Cho GY, et al. Mechanical dyssynchrony assessed by tissue Doppler imaging is a powerful predictor of mortality in congestive heart failure with normal QRS duration. J Am Coll. 2005;46(12):2237-2243.

43. Kashani A, Barold SS. Significance of QRS complex duration in patients with heart failure. J Am Coll Cardiol. 2005;46(12):2183-2192.

44. Vanagt WY, et al. Pacing-induced dys-synchrony preconditions rabbit myocardium against ischemia/reperfusion injury. Circulation. 2006; 114(1 suppl):I264-I269.

45. van Berlo JH, Maillet M, Molkentin JD. Signaling effectors underlying pathologic growth and remodeling of the heart. J Clin Invest. 2013;123(1):37-45.

46. Ambardekar AV, et al. Incomplete recovery of myocyte contractile function despite improvement of myocardial architecture with left ventricular assist device support. Circ Heart Fail. 2011; 4(4):425-432.

47. Fan D, Wannenburg T, de Tombe PP. Decreased myocyte tension development and calcium responsiveness in rat right ventricular pressure overload. Circulation. 1997;95(9):2312-2317.

48. Zhang $\mathrm{P}$, et al. Multiple reaction monitoring to identify site-specific troponin I phosphorylated residues in the failing human heart. Circulation. 2012; 126(15):1828-1837.

49. Pyle WG, Hart MC, Cooper JA, Sumandea MP, de Tombe PP, Solaro RJ. Actin capping protein: an essential element in protein kinase signaling to the myofilaments. Circ Res. 2002;90(12):1299-1306

50. Gupta MP, Samant SA, Smith SH, Shroff SG. HDAC4 and PCAF bind to cardiac sarcomeres and play a role in regulating myofilament contractile activity. J Biol Chem. 2008;283(15):10135-10146.

51. Ponomareva EV, Ogneva IV. [Contractile properties, transversal stiffness and cytoskeletal protein content in Mongolian gerbils soleus fibers under long-term hindlimb suspension]. Biofizika. 2012; 57(4):683-689.

52. Hassel D, et al. Nexilin mutations destabilize cardiac Z-disks and lead to dilated cardiomyopathy. Nat Med. 2009;15(11):1281-1288.

53. Kimura A. Molecular basis of hereditary cardiomyopathy: abnormalities in calcium sensitivity, stretch response, stress response and beyond. J Hum Genet. 2010;55(2):81-90.

54. Kuster DW, et al. GSK3 $\beta$ phosphorylates newly identified site in the proline-alanine-rich region of cardiac myosin-binding protein $\mathrm{C}$ and alters crossbridge cycling kinetics in human: short communication. Circ Res. 2013;112(4):633-639.

55. Kovacic-Milivojevic B, Roediger F, Almeida EA, Damsky CH, Gardner DG, Ilic D. Focal adhesion kinase and $\mathrm{p} 130 \mathrm{Cas}$ mediate both sarcomeric organization and activation of genes associated with cardiac myocyte hypertrophy. Mol Biol Cell. 2001; 12(8):2290-2307.

56. Kong SW, et al. Heart failure-associated changes in RNA splicing of sarcomere genes. Circ Cardiovasc Genet. 2010;3(2):138-146.

57. Roof DJ, Hayes A, Adamian M, Chishti AH, Li T. Molecular characterization of abLIM, a novel actinbinding and double zinc finger protein. J Cell Biol. 1997;138(3):575-588.

58. Liu CP, Ting CT, Lawrence W, Maughan WL, Chang MS, Kass DA. Diminished contractile response to increased heart rate in intact human left ventricular hypertrophy. Systolic versus diastolic determinants. Circulation. 1993;88(4 pt 1):1893-1906. 